\title{
Editorial
}

\section{Quantitative Soil Spectroscopy}

\author{
Sabine Chabrillat, ${ }^{1}$ Eyal Ben-Dor, ${ }^{2}$ Raphael A. Viscarra Rossel, ${ }^{3}$ \\ and José A. M. Demattê $\hat{~}^{4}$ \\ ${ }^{1}$ Section of Remote Sensing, Helmholtz Centre Potsdam, GFZ German Research Centre for Geosciences, \\ Telegrafenberg, 14473 Potsdam, Germany \\ ${ }^{2}$ The Remote Sensing Laboratory, Department of Geography and Human Environment, Tel-Aviv University, \\ P.O. Box 39040, Ramat Aviv, 69978 Tel-Aviv, Israel \\ ${ }^{3}$ Soil and Landscape Program, CSIRO Land and Water, Bruce E. Butler Laboratory, Clunies-Ross Street Black Mountain, \\ P.O. Box 1666, Canberra, ACT 2601, Australia \\ ${ }^{4}$ Soil Science Department, Luiz de Queiroz College of Agriculture, São Paulo, University of Piracicaba, SP 13418-900, Brazil
}

Correspondence should be addressed to Sabine Chabrillat; chabri@gfz-potsdam.de

Received 13 January 2013; Accepted 13 January 2013

Copyright (C) 2013 Sabine Chabrillat et al. This is an open access article distributed under the Creative Commons Attribution License, which permits unrestricted use, distribution, and reproduction in any medium, provided the original work is properly cited.

Interest in the use of visible-near infrared reflectance spectroscopy for the determination of mineralogical composition in soils and planetary surfaces has been demonstrated since the 1970s with the development of databases of minerals spectra recorded in the laboratory by Hunt and Salisbury. A little later, in the early 1980 s, the first spectral database (or library) of American soils was generated by Stoner and Baumgardner. In the mid-1980s, several workers demonstrated that different soil attributes could be estimated from the spectral reflectance measurement and the quantitative era of soil spectroscopy begun. The attractiveness of soil spectroscopy is that measurements are rapid and estimates of soil properties are inexpensive compared to conventional soil analyses. Nowadays, research on quantitative soil spectroscopy for the prediction of soil properties, prompted by developments in multivariate statistics and chemometrics, is continuing to grow. Over the past decades, the availability of new high signal-to-noise ratio hyperspectral sensors that can be mounted on airborne platforms or that can be used in the laboratory and the field opened significant new possibilities toward the quantitative analyses of the physical and biochemical composition of the Earth's soil.

The spectral reflectance of soil is a cumulative property that derives from the inherent spectral behaviour of heterogeneous combinations of minerals, organic matter, and water molecules in the soil. Studies on soil spectroscopy relate primarily to the following attributes. (i) Soil water, a key variable in hydrologic cycle, controls processes such as infiltration and discharge with consequences for plant growth, soil erosion, and land degradation. (ii) Soil carbon (content and composition), through its key role in the carbon cycle, is an important variable in global climate models. Soil organic matter, of which carbon is a major part, holds a large proportion of nutrients, cations, and trace elements that are needed for plant growth. (iii) Soil mineralogy and texture are important soil properties as they affect physical, chemical, and biological soil processes. Other soil parameters might also be estimated through either direct or indirect relationships of soil reflectance with the chemical, physical, and biological characteristics of the soil matrix.

In this regard, optical and infrared sensing covering the visible, infrared, and thermal parts of the electromagnetic spectrum, respectively, have shown good potential for retrieving information on soil attributes. Across this spectral range, three regions sensitive to soil properties can be defined as follows:

(i) The visible-near infrared (VNIR) spectral region from 0.4 to $1 \mu \mathrm{m}$ contains information on soil color, iron content and composition, soil water, and organic matter.

(ii) The near infrared (NIR) which also referred as the short-wave infrared (SWIR) in remote sensing from 
1 to $2.5 \mu \mathrm{m}$ contains information on phyllosilicates, most sorosilicates, hydroxides, some sulphates, amphiboles, carbonates, soil water, and organic matter. A number of bench top and portable spectrometers combine the VNIR and the SWIR to provide spectra in the vis-NIR range from 0.4 to $2.5 \mu \mathrm{m}$.

(iii) The mid-infrared (mid-IR), which is also referred to as the thermal infrared (TIR) in remote sensing, ranges from 2.5 to $25 \mu \mathrm{m}$ including the medium (MWIR) and long-wave (LWIR) infrared spectral regions (3-5 and 8-14 $\mu \mathrm{m})$ that are atmospheric windows. This spectral region contains information on quartz, feldspars, silicate minerals, mafic, clay, carbonate mineral group, and organic compounds.

While the chemical attributes influencing soil reflectance are based on absorption of radiation by chemical compounds in selected frequencies, a soil spectrum is also affected by physical characteristics of the soil such as particle size, surface roughness and soil water, which will be dominated by scattering radiation across all spectral regions.

Different methods have been proposed for the quantification of soil properties using spectra. For instance, methods based on the analytical and physical characteristics of the signal and empirical methods based on chemometrics have been shown to have good effective predictability. Therefore, regressions can be developed using field and laboratory data for calibration, allowing soil reflectance to be related with soil properties. Direct relationships between specific absorption features with soil mineralogy and water content are the more commonly used methods, particularly in remote sensing. However, methods that use empirical multivariate relationships of reflectance with soil properties are nowadays often developed in remote sensing to help enlarge the prediction envelope of quantitative soil spectroscopy toward more soil attributes.

These advances of soil spectroscopy have a significant impact in many soil science fields; for instance, quantitative spectroscopy is being used in the evaluation and monitoring of soil quality and soil function (e.g., water storage and carbon storage), soil fertility and soil threats (e.g., acidification and erosion), and soil pedogenesis. For example, soil degradation (salinity, erosion, and deposition), soil mapping and classification, soil genesis and formation, soil contamination, and soil hazards (swelling soils) are important issues that are nowadays examined using hyperspectral remote sensing, enlarging the soil spectroscopy into a spatial domain. Indeed, quantitative soil spectroscopy is being used to collect the large amounts of data needed for digital soil mapping and is used as a support in many activities toward future global soil monitoring. In this sense, the upcoming future availability of high signal-to-noise ratio satellite imaging spectrometers such as EnMAP (German satellite initiative, in phase D), PRISMA (Italian satellite initiative, prephase A), HYPXIM (French satellite initiative, phase A will start in the end of 2012), SHALOM (Italy-Israel initiative), and HyspIRI (USA satellite initiative, prephase A) will be a major step toward the operational quantitative monitoring of soil surfaces over large areas. Then, hyperspectral remote sensing that senses the upper surface is used together with on-ground (or proximal) soil spectroscopy for global soil characterization and monitoring.

In this context, the main focus of this special issue is to present current research on soil applications of reflectance spectroscopy at both point and imagery domains, illustrating state-of-the-art methods in quantitative soil spectroscopy. Our principal objective is that this issue demonstrates the potential of soil spectroscopy for the quantitative description of spatial distribution of soils and their properties. The special issue received 13 papers from all over the world covering diverse topics. Of those, after a peer review process, 8 were considered for publication.

M. L. McDowell et al. discuss the effects of subsetting by carbon content, soil order, and spectral classification for the prediction of soil total carbon using the VNIR, SWIR, and TIR $(0.4-14 \mu \mathrm{m})$ spectral regions associated with partial least squares regression (PLSR) models based on diffuse reflectance information received by a point spectrometer. The soil samples originated from the Hawaiian agricultural lands with very low to high carbon content. The results show that different subsetting methods explored lead to various results. Subsetting of only low carbon content samples showed improvement in the prediction of carbon content.

R. D. Hewson et al. evaluate the ability of SWIR and TIR (2-14 $\mu \mathrm{m})$ spectral regions to characterize soil composition and texture using particle separated soil samples from Tick Hill, QLD, Australia, and natural soils from the United States Department of Agriculture (ASTER spectral library). Derivation of clay mineral content, quartz content, and organic carbon content is performed based on indices from the TIR region using the $9.5 \mu \mathrm{m}, 8.62 \mu \mathrm{m}$, and $3.45 \mu \mathrm{m}$ spectral features, respectively. A good correlation with mineral content was observed for the quartz index, which demonstrated the added value of the TIR region (emissivity) to the VNIR-SWIR region (reflectance).

H. Buddenbaum et al. explore the influence of several spectral pretreatments on chemometric analyses of soil profiles on a submillimetre scale. Two $30 \mathrm{~cm}$ deep cores from southern German soils were measured in the laboratory with a hyperspectral scanner (HySpex-1600) covering the VNIR spectral range $(0.4-1 \mu \mathrm{m})$. The results showed that preprocessing methods have a minor influence on the PLS regression results for the estimation of elemental concentrations in the soil cores.

H. Gerighausen et al. spatially estimate clay and organic carbon content in agricultural soils using multiannual imaging spectroscopy data (HyMap: 0.4-2.4 $\mu \mathrm{m}$ ). The study area is the test site of Durable Environmental Multidisciplinary Monitoring Information Network (DEMMIN) in Germany, with low average organic carbon content. PLS regressions are used to test the predictive ability of the models for parameter prediction for the different years of hyperspectral survey.

A. Bayer et al. compare between two approaches for the mapping and quantification of soil organic carbon, iron oxides, and clay content from hyperspectral imagery (HyMap: 0.4-2.4 $\mu \mathrm{m}$ ) over large areas in the Thicket Biome, South Africa. A physical approach based on a set of diagnostic spectral features linked with chemical reference data 
using multiple linear regression techniques is developed and compared with results from multivariate method such as PLS regression models.

N. Goldshleger et al. study the effect of raindrop energy on the water infiltration and runoff into the soil profile and on the soil surface SWIR reflectance (1.2-2.4 $\mu \mathrm{m})$ by studying seven soils from Israel and the USA subjected to artificial (controlled) rainstorm events. The spectral properties of crust formed on the soil surface were analyzed by using a nonlinear artificial neural network (ANN) method and compared with PLS regression. The ANN technique provides better results for the correlation of spectral reflectance with infiltration rate.

H. Bartholomeus et al. investigate the estimation of soil properties in Siberian Tundra soils using reflectance information and study the relationships with the plant species composition. PLS and stepwise multiple linear (SML) regression models derived from the reflectance measurements are used to proximate total C, N, pH, K, and P in the soil. Subsequently, SML regressions yielded high accuracy models for prediction of $\mathrm{C}$ and N, and PLS models yielded good prediction model for $\mathrm{K}$ and moderate model for $\mathrm{pH}$.

G. Schwartz et al. propose the use of reflectance spectroscopy method as an alternative tool for detecting contamination of total petroleum hydrocarbons in the soils. They compare the spectral method with three commercial certified laboratory analyses using traditional methods. The results show that reflectance spectroscopy provides a similar accuracy to commercial laboratories method and could be used for initial field-screening investigations holding cost-effective and rapid assessment.

We hope that you find the special issue interesting and useful and that it will act as a precursor for more studies to come in soil spectroscopy.

\section{Acknowledgments}

We thank the EU FP7 EUFAR Project for supporting the Expert Working Group (EWG) in soil applications of hyperspectral imagery and the organization of the first and second workshops of the EWG held at Potsdam, Germany, on April 2010 and August 2011, bringing together for the first time the community on soil spectroscopy. This project is further supported by the ISPRS Technical Group VII/3 "Information extraction from hyperspectral data." Our thanks also to all the reviewers for their timely reports and constructive comments and the staff of the Editorial Office of Applied and Environmental Soil Science for their support. S. Chabrillat wishes to thank the EnMAP Satellite Project funded by $\mathrm{BMBF} / \mathrm{BMWi}$ and the GFZ for additional support. R. A. Viscarra Rossel's contribution was funded by the CSIRO's Sustainable Agriculture Flagship (SAF).

Sabine Chabrillat Eyal Ben-Dor Raphael A. Viscarra Rossel José A. M. Demattê 

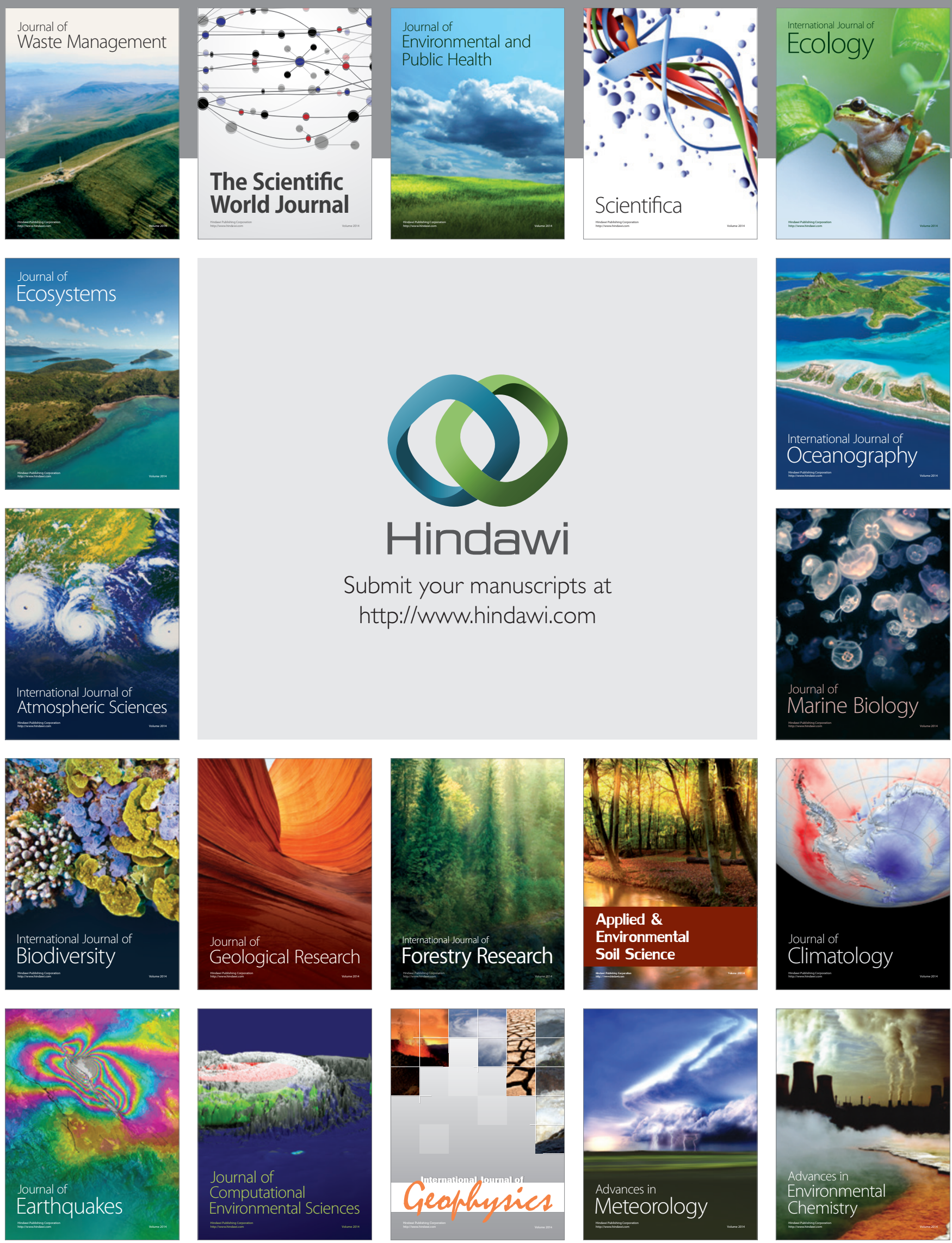